\title{
Phenotypic screen identifies JAK2 as a major regulator of FAT10 expression
}

Nava Reznik ${ }^{1,2}$, Noga Kozer ${ }^{3}$, Avital Eisenberg-Lerner ${ }^{1}$, Haim Barr ${ }^{3}$, Yifat Merbl ${ }^{1,{ }^{*}}$, Nir London ${ }^{2, *}$

${ }^{1}$ Department of Immunology, The Weizmann Institute of Science, Rehovot, 7610001, Israel.

${ }^{2}$ Department of Organic Chemistry, The Weizmann Institute of Science, Rehovot, 7610001, Israel.

${ }^{3}$ Wohl Institute for Drug Discovery of the Nancy and Stephen Grand Israel National Center for Personalized Medicine, The Weizmann Institute of Science, Rehovot, 7610001, Israel.

* Corresponding authors:

Yifat Merbl yifat.merbl@weizmann.ac.il

Nir London nir.london@weizmann.ac.il

Keywords: FAT10, JAK2, phenotypic screen, AZ960, Ubiquitin-like modifiers 


\section{Supplementary tables}

Table S1 - Pathway enrichment analysis of all targets of identified hit compounds (PANTHER Classification System).

\begin{tabular}{|c|c|c|}
\hline PANTHER Pathways & $\begin{array}{l}\text { Fold } \\
\text { Enrichment }\end{array}$ & $\begin{array}{l}\text { Raw P- } \\
\text { value }\end{array}$ \\
\hline JAK/STAT signaling pathway (P00038) & 39.84 & $6.27 \mathrm{E}-06$ \\
\hline Insulin/IGF pathway-protein kinase B signaling cascade (P00033) & 35.28 & 1.38E-12 \\
\hline p53 pathway feedback loops 2 (P04398) & 32.77 & $1.57 \mathrm{E}-14$ \\
\hline Ras Pathway (P04393) & 32.03 & $1.22 \mathrm{E}-16$ \\
\hline p53 pathway (P00059) & 31.95 & $2.44 \mathrm{E}-23$ \\
\hline p53 pathway by glucose deprivation (P04397) & 29.45 & 1.79E-05 \\
\hline Interferon-gamma signaling pathway (P00035) & 28.22 & $1.81 \mathrm{E}-06$ \\
\hline Hypoxia response via HIF activation (P00030) & 26.46 & $2.41 \mathrm{E}-06$ \\
\hline PDGF signaling pathway (P00047) & 25.79 & $7.91 \mathrm{E}-25$ \\
\hline PI3 kinase pathway (P00048) & 22.96 & 5.49E-09 \\
\hline Interleukin signaling pathway (P00036) & 22.33 & $9.50 \mathrm{E}-13$ \\
\hline VEGF signaling pathway (P00056) & 21.46 & 1.01E-09 \\
\hline B cell activation (P00010) & 21.17 & 1.13E-09 \\
\hline $\begin{array}{l}\text { Insulin/IGF pathway-mitogen activated protein kinase kinase/MAP } \\
\text { kinase cascade (P00032) }\end{array}$ & 19.92 & 7.16E-05 \\
\hline Axon guidance mediated by netrin (P00009) & 18.81 & 8.78E-05 \\
\hline Angiogenesis (P00005) & 18.61 & 3.99E-19 \\
\hline Parkinson disease (P00049) & 18.08 & $7.03 E-11$ \\
\hline 5-Hydroxytryptamine degredation (P04372) & 16.93 & 7.31E-03 \\
\hline EGF receptor signaling pathway (P00018) & 15.95 & $5.25 \mathrm{E}-12$ \\
\hline Toll receptor signaling pathway (P00054) & 15.87 & $3.50 \mathrm{E}-06$ \\
\hline CCKR signaling map (P06959) & 15.81 & $2.80 \mathrm{E}-21$ \\
\hline FGF signaling pathway (P00021) & 15.52 & $3.19 \mathrm{E}-10$ \\
\hline Axon guidance mediated by semaphorins (P00007) & 14.11 & $1.01 \mathrm{E}-02$ \\
\hline Apoptosis signaling pathway (P00006) & 13.88 & 5.75E-09 \\
\hline FAS signaling pathway (P00020) & 12.7 & $2.05 \mathrm{E}-03$ \\
\hline T cell activation (P00053) & 12.35 & $2.52 \mathrm{E}-06$ \\
\hline Oxidative stress response (P00046) & 12.09 & $4.28 \mathrm{E}-04$ \\
\hline Integrin signalling pathway (P00034) & 11.17 & $3.33 \mathrm{E}-10$ \\
\hline Endothelin signaling pathway (P00019) & 8.91 & $3.10 \mathrm{E}-04$ \\
\hline Dopamine receptor mediated signaling pathway (P05912) & 8.61 & $5.81 \mathrm{E}-03$ \\
\hline Cadherin signaling pathway (P00012) & 8.57 & $6.25 \mathrm{E}-06$ \\
\hline Alzheimer disease-amyloid secretase pathway (P00003) & 7.78 & 2.04E-03 \\
\hline $\begin{array}{l}\text { Inflammation mediated by chemokine and cytokine signaling } \\
\text { pathway (P00031) }\end{array}$ & 7.53 & 1.07E-07 \\
\hline $\begin{array}{l}\text { Heterotrimeric G-protein signaling pathway-Gi alpha and Gs alpha } \\
\text { mediated pathway (P00026) }\end{array}$ & 5.26 & 1.15E-03 \\
\hline Gonadotropin-releasing hormone receptor pathway (P06664) & 5.13 & 8.27E-05 \\
\hline Alzheimer disease-presenilin pathway (P00004) & 3.81 & 1.10E-02 \\
\hline Wnt signaling pathway (P00057) & 3.73 & 3.11E-03 \\
\hline
\end{tabular}




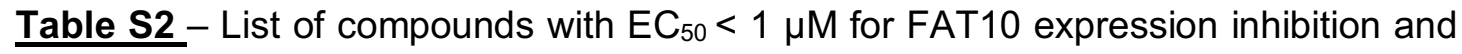
no effect on cell viability.

\begin{tabular}{|c|c|c|c|}
\hline Compound & $\begin{array}{c}\text { Annotated } \\
\text { target }\end{array}$ & $\begin{array}{c}\text { EC }_{50}(\boldsymbol{\mu M}) \\
\text { FAT10 }\end{array}$ & $\mathbf{R}^{\mathbf{2}}$ \\
\hline Panobinostat & HDAC & $<0.03$ & 0.789 \\
\hline KX2-391 & SRC & 0.035 & 0.940 \\
\hline Selinexor (KPT-330) & XPO1 & 0.049 & 0.998 \\
\hline BI 2536 & PLK1 & 0.052 & 0.911 \\
\hline (+)-JQ1 & BRD4 & 0.067 & 0.993 \\
\hline XMD8-92 & MAPK7 & 0.075 & 0.885 \\
\hline AZ960 & JAK2 & 0.123 & 0.977 \\
\hline TPCA-1 & IKBKB & 0.203 & 0.980 \\
\hline Ruxolitinib (INCB018424) & JAK1/2 & 0.256 & 0.780 \\
\hline CEP33779 & JAK2 & 0.299 & 0.984 \\
\hline TG101348 (SAR302503) & JAK2 & 0.357 & 0.988 \\
\hline Tivantinib (ARQ 197) & MET & 0.388 & 0.925 \\
\hline Cyt387 & JAK1/2 & 0.465 & 0.983 \\
\hline Afatinib (BIBW2992) & EGFR & 0.473 & 0.964 \\
\hline SKI II & SPHK1 & 0.507 & 0.963 \\
\hline Rebastinib (DCC-2036) & ABL1 & 0.564 & 0.554 \\
\hline BAY 61-3606 & SYK & 0.656 & 0.929 \\
\hline Ki16198 & LPAR1/3 & 0.661 & 0.913 \\
\hline BIX 01294 & EHMT2 & 0.738 & 0.903 \\
\hline Neratinib (HKI-272) & EGFR/ERBB2 & 0.743 & 0.930 \\
\hline Tofacitinib (CP-690550) & JAK3 & 0.745 & 0.812 \\
\hline & & & \\
\hline
\end{tabular}




\section{Supplementary figures}

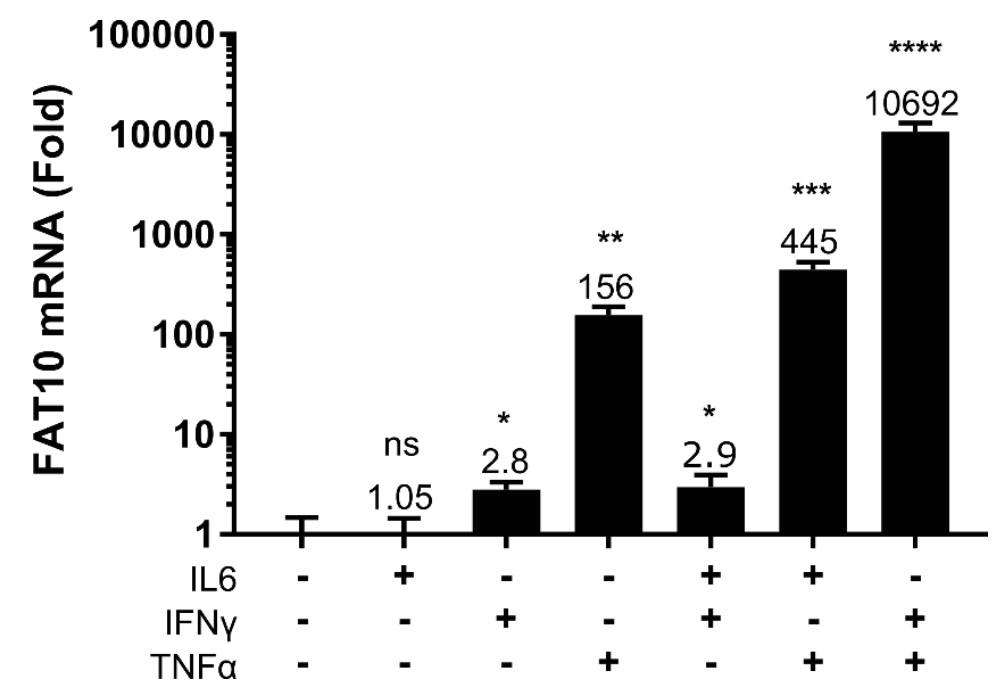

Figure S1. Different combinations of cytokine treatment result in different extent of FAT10 transcription. qPCR analysis of FAT10 mRNA levels in HEK293 treated with either IL6, IFNy and TNFa alone or in combination. Cells were harvested after total of $48 \mathrm{~h}$ cytokines treatment. Significance calculated compared to untreated cells. ns $=$ non-significant, ${ }^{*}=\mathrm{P} \leq 0.05,{ }^{* *}=\mathrm{P} \leq 0.01,{ }^{* * *}=\mathrm{P} \leq 0.001,{ }^{* * * *}=\mathrm{P} \leq 0.0001$. 
A

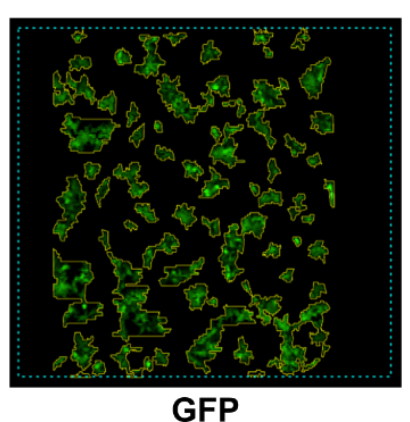

B

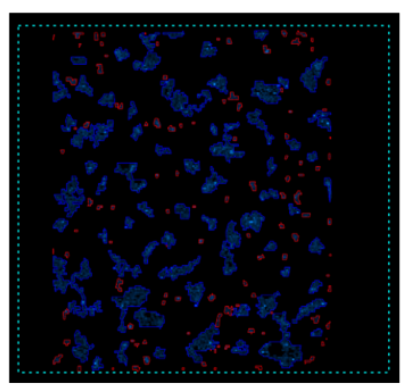

Hoechst

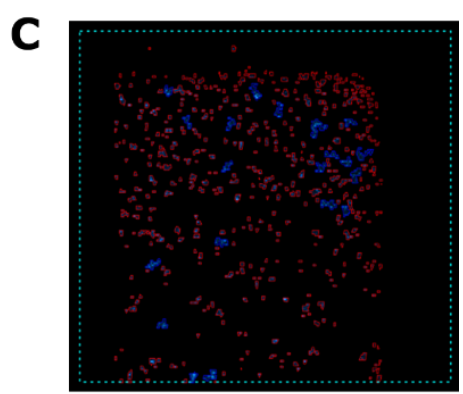

Hoechst

Figure S2. Representative images of control wells taken by laser scanning imaging cytometer. A. - B. DMSO and TI treated well. A. GFP signal, fluorescent objects identified are marked by a yellow line. B. Hoechst signal, objects defined as "Live" are marked by a blue line. C. Well treated with 10\% DMSO, demonstrates dead cells control. Objects circled in red defined as "Dead" cells. 

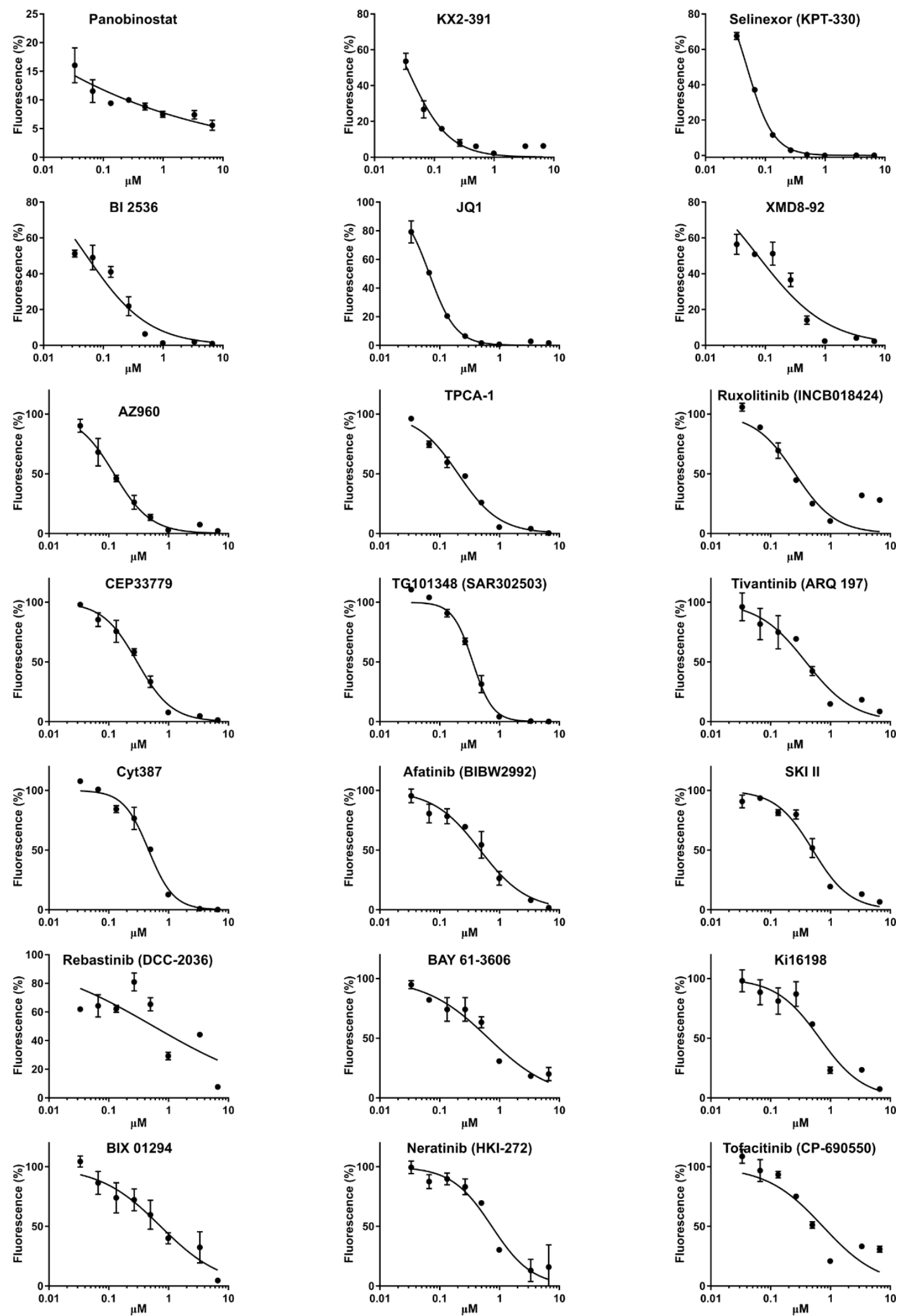

Figure S3. Dose response curves for 21 compounds with $\mathrm{EC}_{50}<1 \mu \mathrm{M}$ for FAT10 expression inhibition. 

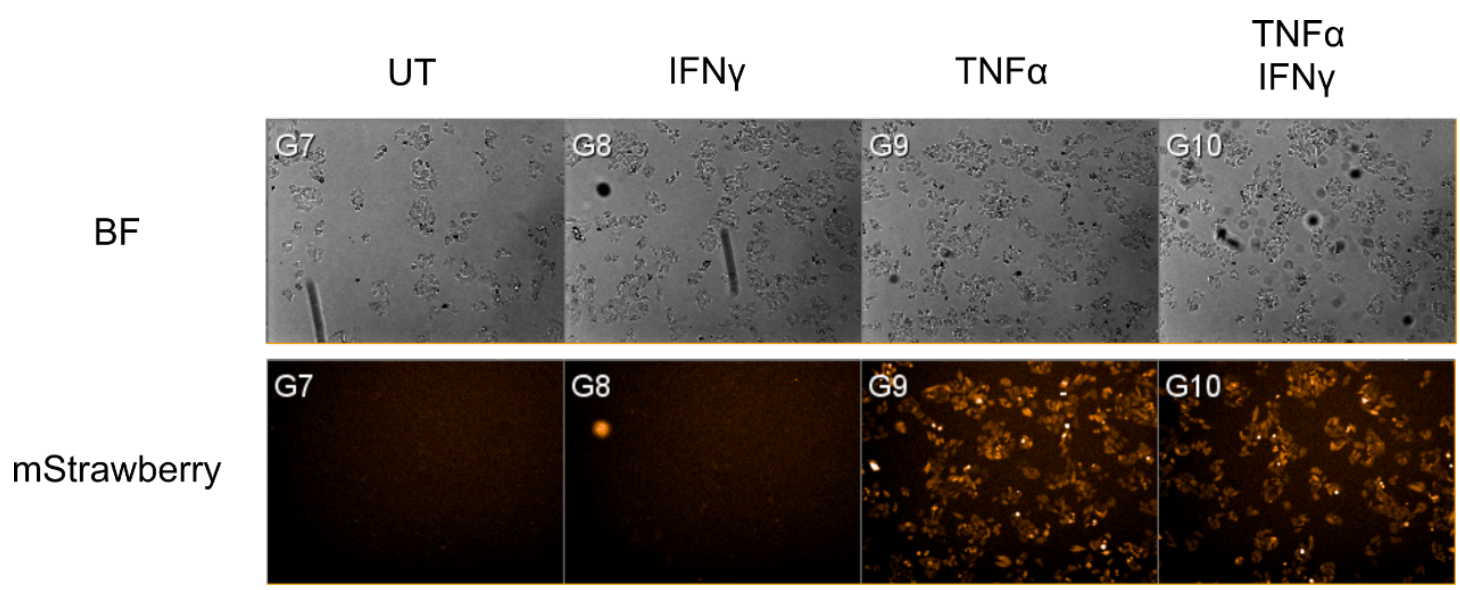

Figure S4. Representative images of NFKB reporter cell line A375 treated with IFNy and TNF $\alpha$ for $24 h$. 

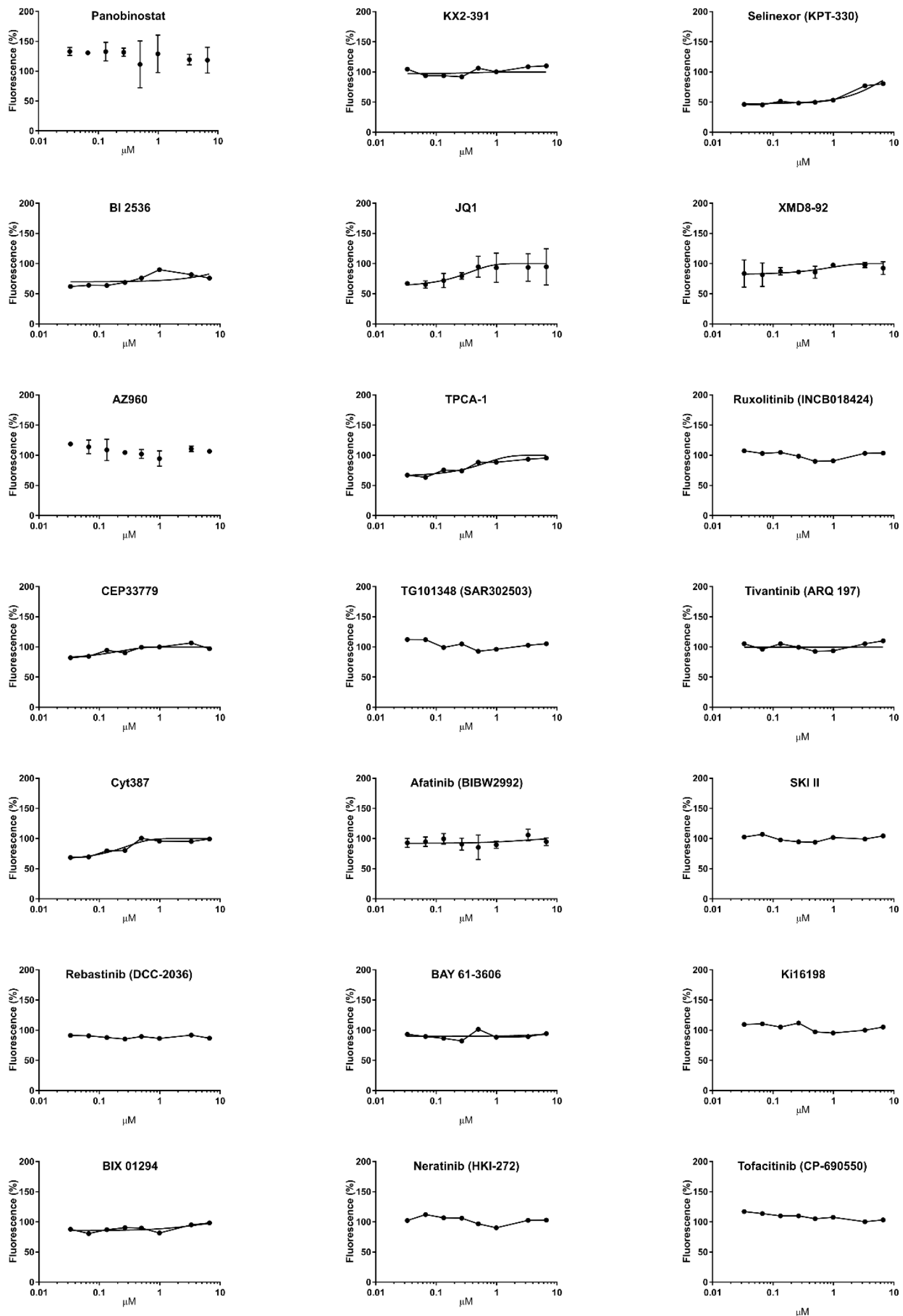

Figure S5. Dose response curves for the 21 FAT10 active compounds assessing $\mathrm{NF}$ KB inhibition using an NFKB reporter cell line. 


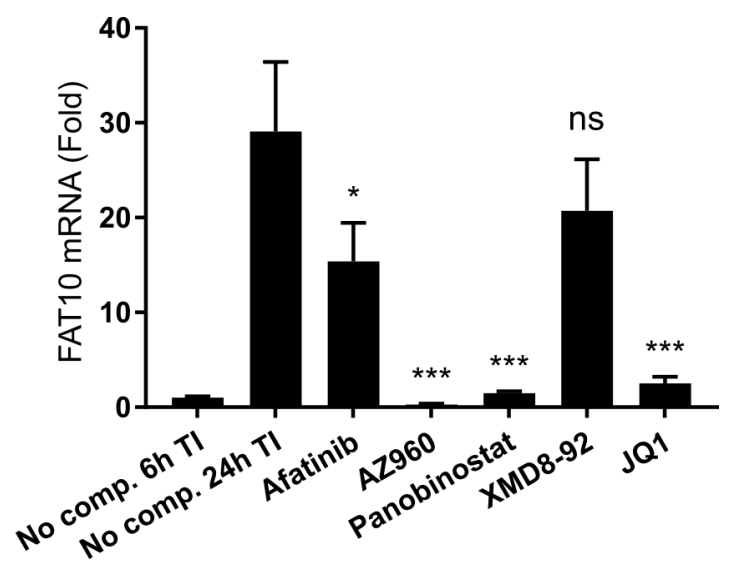

Figure S6. Short pre-incubation with pro-inflammatory cytokines does not rescue down regulation by screening hits. qPCR analysis of FAT10 mRNA levels upon treatment with 5 selected compounds after $6 \mathrm{~h}$ pre-induction with TI. HEK293 cells were treated with $\mathrm{Tl}$ for $6 \mathrm{~h}$, then $1 \mu \mathrm{M}$ compounds were added and cells were harvested after total $24 \mathrm{~h}$ of TI treatment. FAT10 mRNA levels were normalized to Actin. Significance is calculated in comparison to cells treated with $\mathrm{TI}$ and no compound. 
A

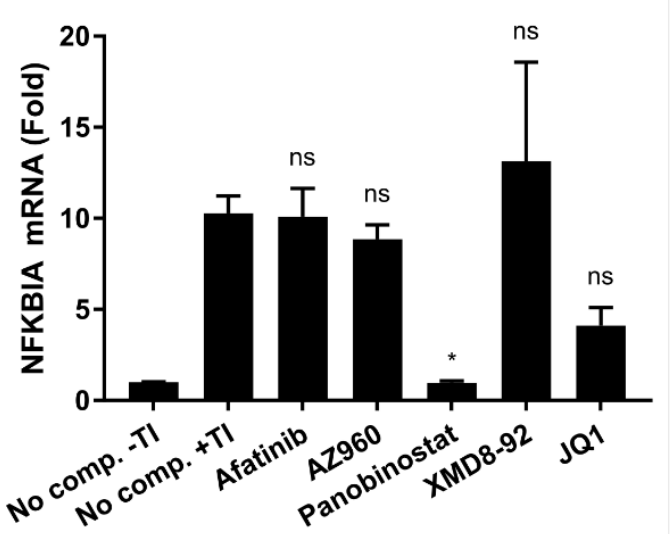

$\mathbf{C}$

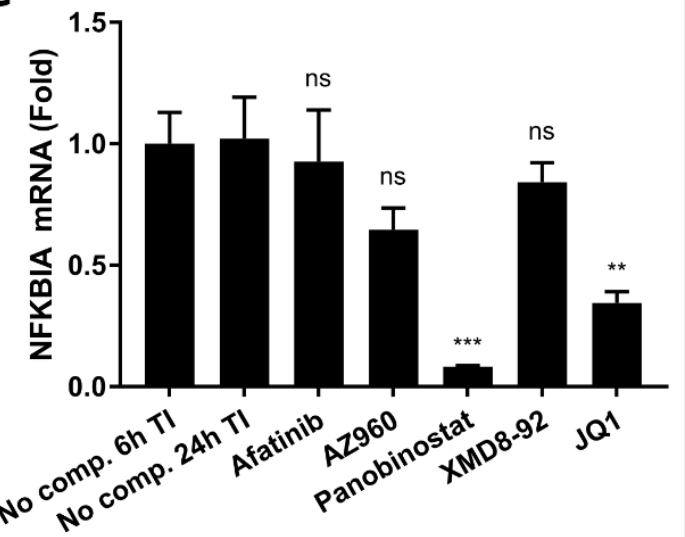

B

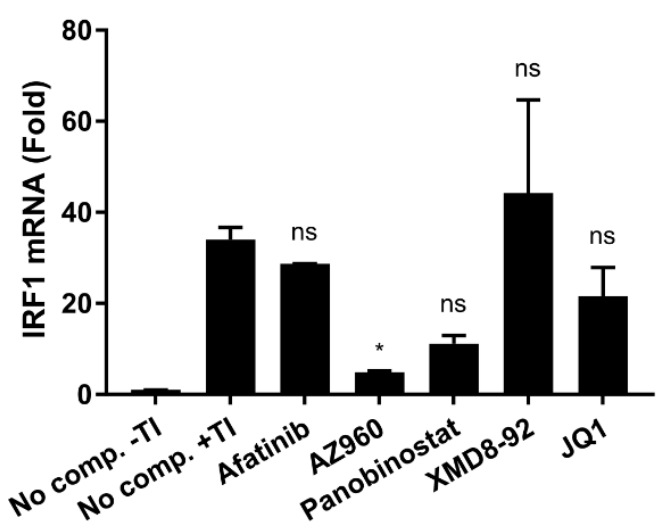

D

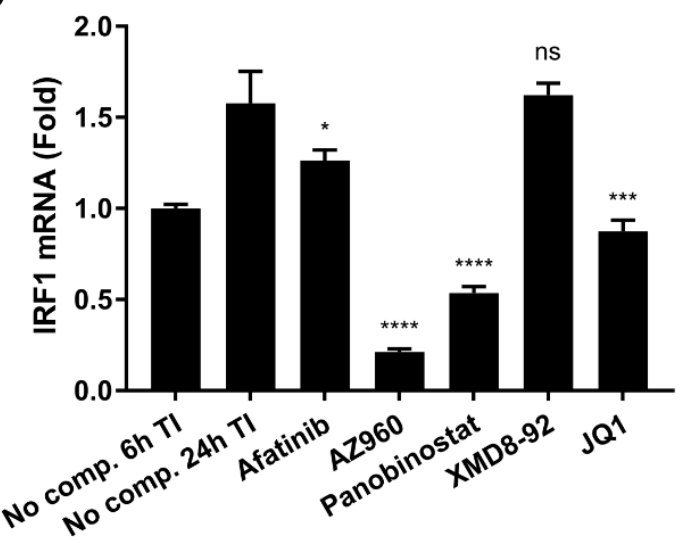

Figure S7. Screening hits affect either NFKB or IFN $\gamma$ signaling pathways. A-B. qPCR analysis of NFKBIA and IRF1 mRNA levels upon treatment with 5 selected compounds. HEK293 cells were pre-incubated $2 \mathrm{~h}$ with $1 \mu \mathrm{M}$ compounds, then treated with $\mathrm{TI}$ and harvested after $24 \mathrm{~h}$ from induction with $\mathrm{TI}$. mRNA levels were normalized to Actin. Significance is calculated in comparison to cells treated with $\mathrm{TI}$ and no compound. C-D. qPCR analysis of NFKBIA and IRF1 mRNA levels upon treatment with 5 selected compounds after $6 \mathrm{~h}$ pre-induction with $\mathrm{TI} .1 \mu \mathrm{M}$ compounds were added and cells were harvested after total $24 \mathrm{~h}$ of TI treatment. mRNA levels were normalized to Actin. Significance is calculated in comparison to cells treated with $\mathrm{TI}$ and no compound. 


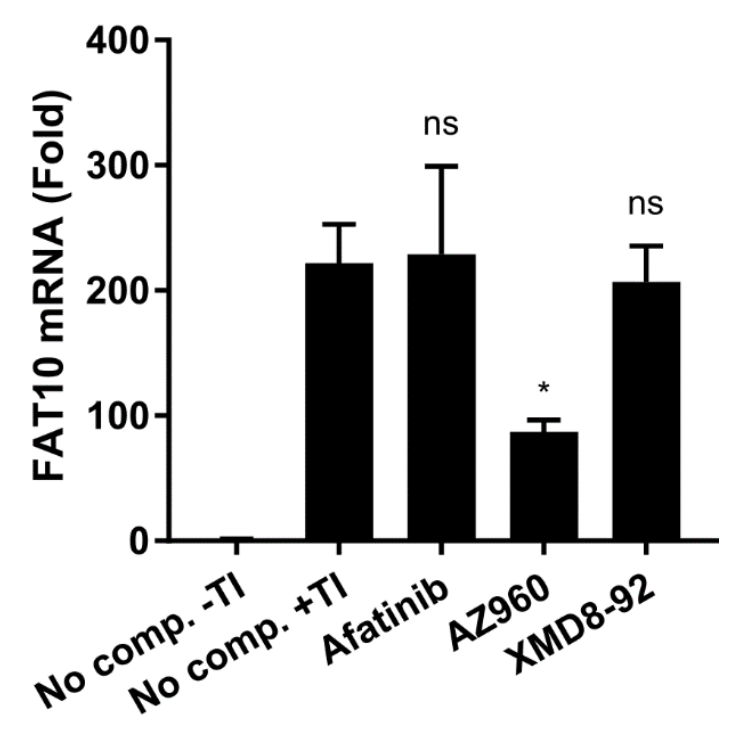

Figure S8. qPCR analysis of FAT10 mRNA levels upon pre-treatment with TI for $24 \mathrm{~h}$, then replacing the media and adding the compounds $(1 \mu \mathrm{M})$ for $24 \mathrm{~h}$. Significance is calculated in comparison to cells treated with $\mathrm{TI}$ and no compound. 
A

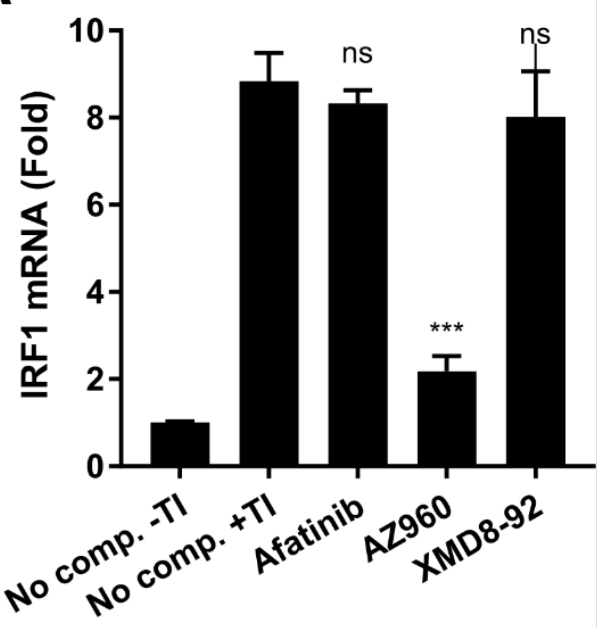

B

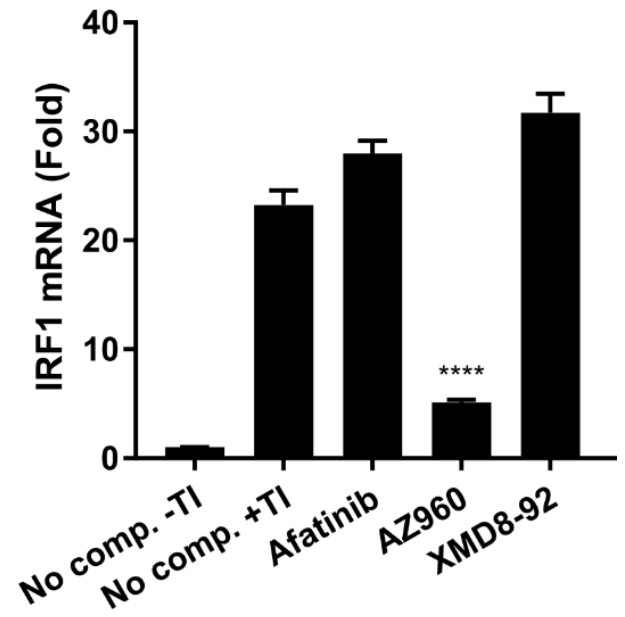

Figure S9. AZ960 inhibits IRF1 expression under pro-inflammatory cytokine induction. qPCR analysis of IRF1 mRNA levels. A. Cells were treated with TI for 24h, then media was replaced, and compounds were added for 24h. Significance is calculated in comparison to cells treated with $\mathrm{Tl}$ and no compound. B. Cells were pretreated with $\mathrm{TI}$ for $24 \mathrm{~h}$, then compounds were added for additional $24 \mathrm{~h}$, total $48 \mathrm{~h} \mathrm{TI}$ treatment. Significance is calculated in comparison to cells treated with $\mathrm{TI}$ and no compound. 

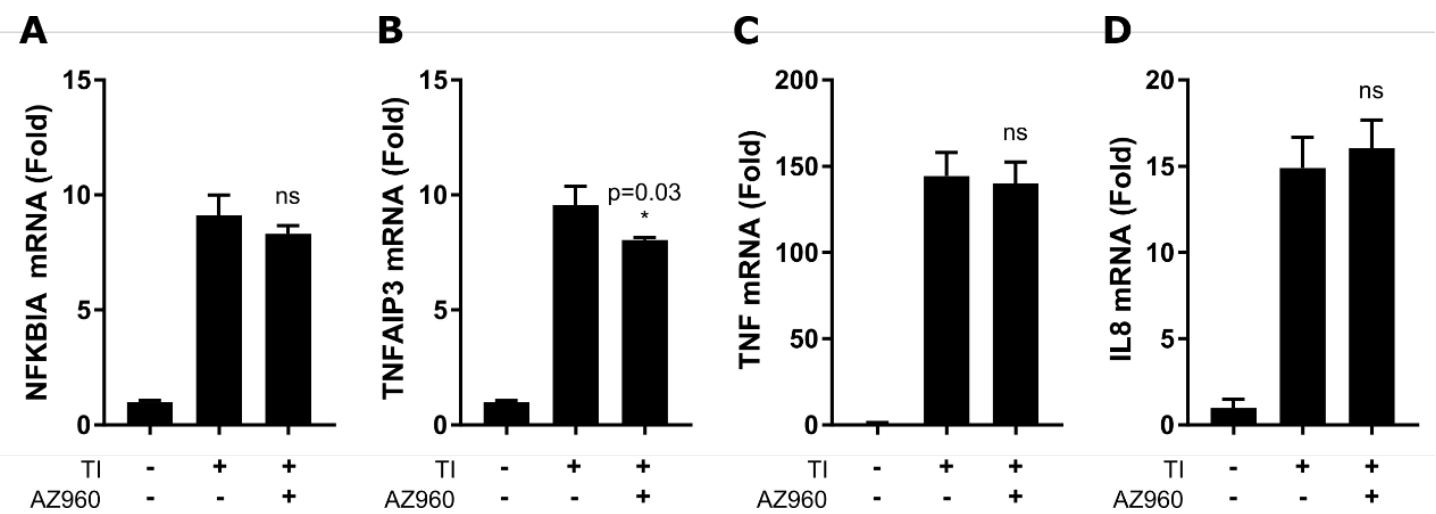

Figure S10. AZ960 does not affect NFKB signaling pathway. QPCR analysis of NFKB downstream target genes. Cells were pre-treated with $\mathrm{TI}$ for $24 \mathrm{~h}$, then incubated with AZ960 at $250 \mathrm{nM}$ for additional $24 \mathrm{~h}$ and harvested after total $48 \mathrm{~h}$ TI treatment. mRNA levels of the following genes were analyzed A. NFKBIA, B. TNFAIP3, C. TNF, and D. IL8. Significance is calculated in comparison to cells treated with TI without AZ960. 
A

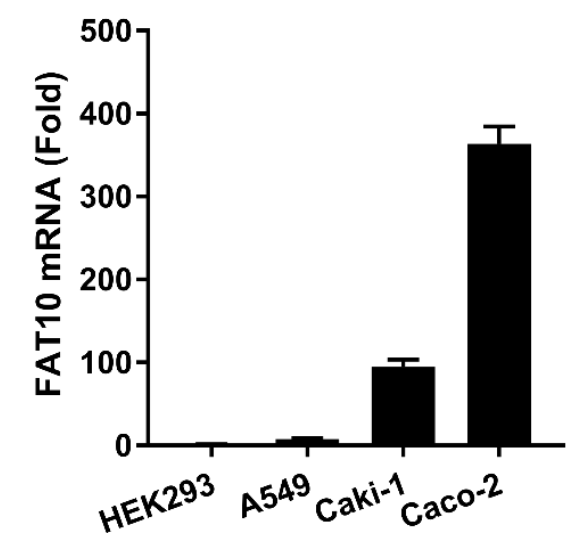

B

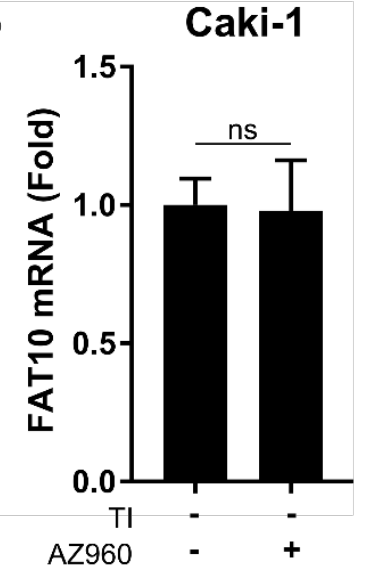

Caco-2

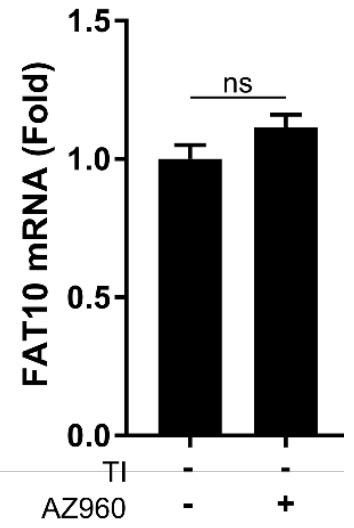

Figure S11. AZ960 does not affect FAT10 levels in absence of TI stimulation. A. qPCR analysis of basal FAT10 mRNA levels of HEK293, A549, Caki-1 and Caco-2 cell lines. B. qPCR analysis of FAT10 mRNA levels of Caki-1 (left panel) and Caco-2 cells (right panel) treated with AZ960 250nM for 24h without TI induction. 

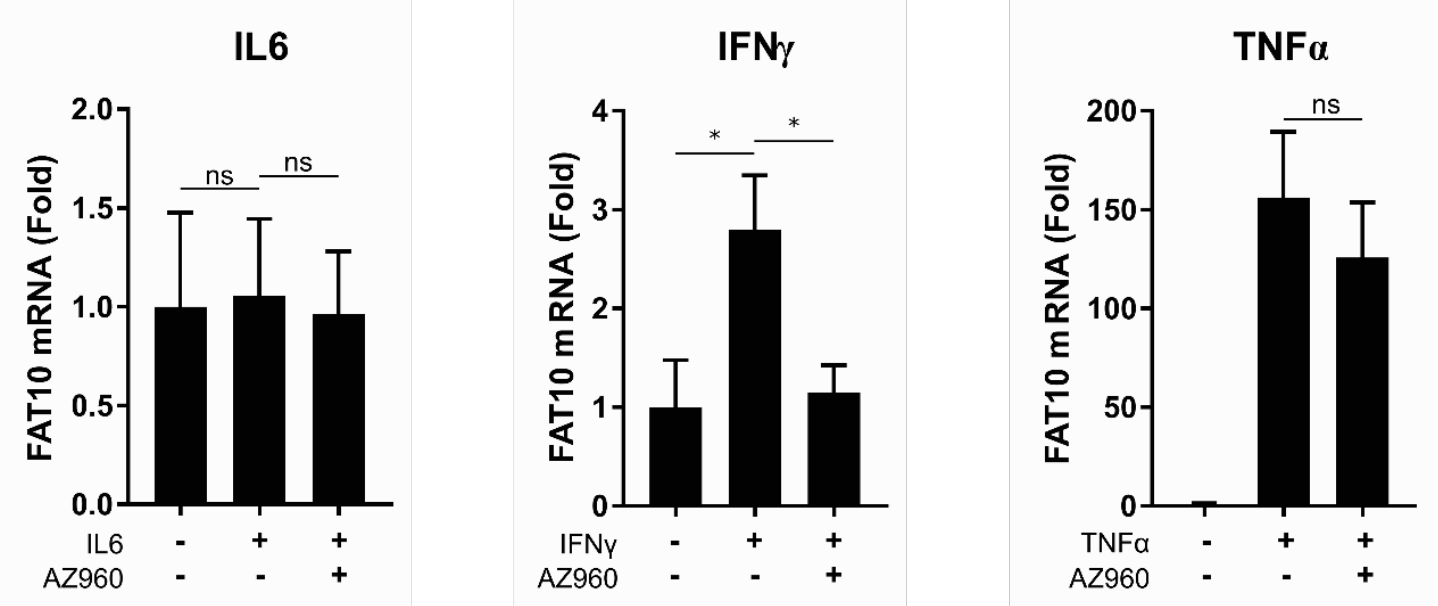

Figure S12. Effect of AZ960 treatment upon single cytokine induction. qPCR analysis of FAT10 mRNA levels in HEK293 treated with either IL6, IFNy or TNF $\alpha$ alone for $24 \mathrm{~h}$ after which AZ960 (250nM) was added for another 24h. Cells were harvested after total of $48 \mathrm{~h}$ cytokines treatment. Part of the data presented also in Figure S1. 
Figure S13. Whole membranes for Figure 4E. Western blot analysis of STAT1(A), pSTAT1(B), STAT3(C), pSTAT3(D), STAT5(E), pSTAT5(F) and FAT10(G) of HEK293 cells treated with $\mathrm{Tl}$ for $24 \mathrm{~h}$, then with AZ960 (25nM and 250nM) for additional $24 \mathrm{~h}$. Cells were harvested after total $48 \mathrm{~h}$ TI treatment.

\section{A. Total STAT1}
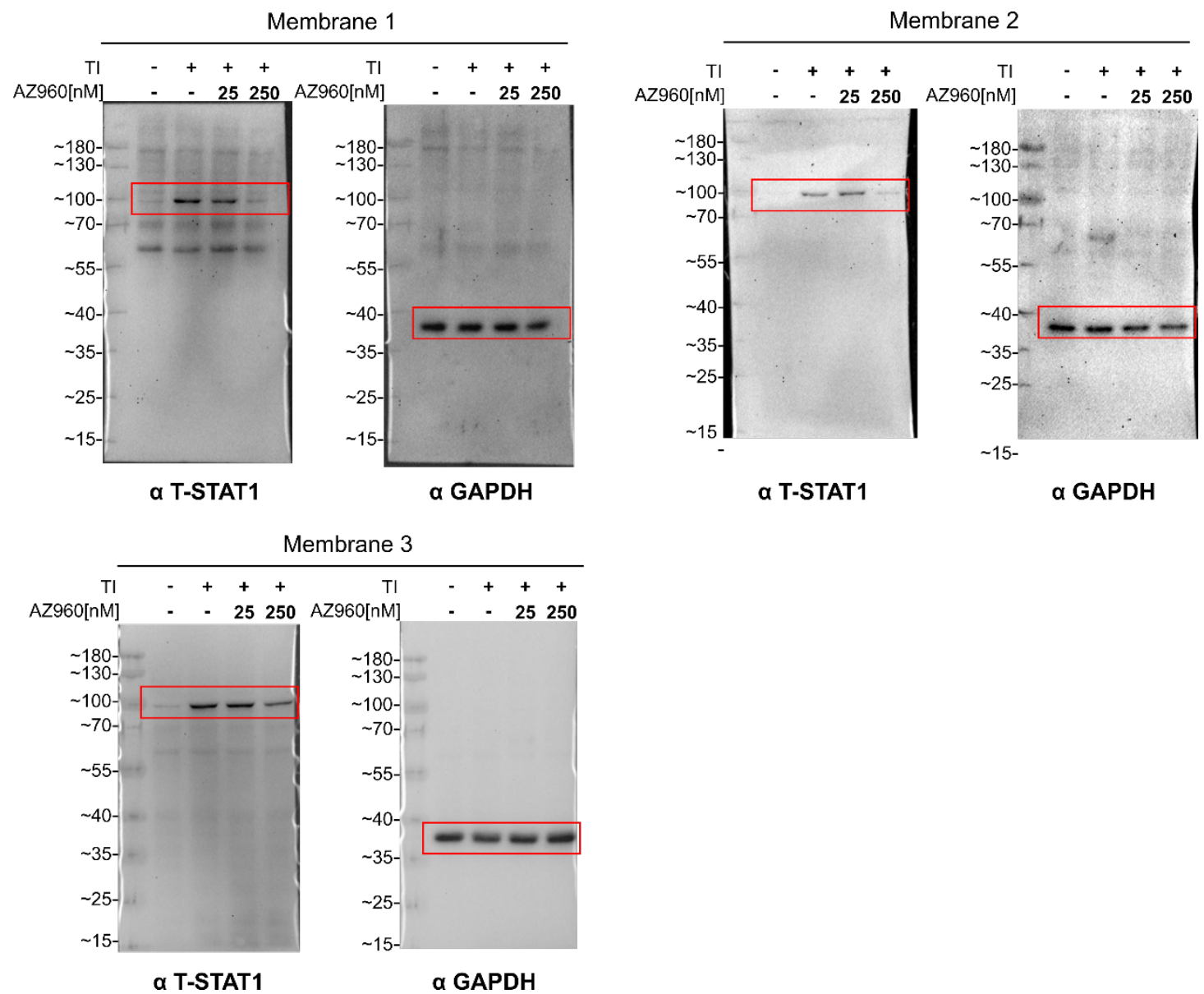
B. p-STAT1
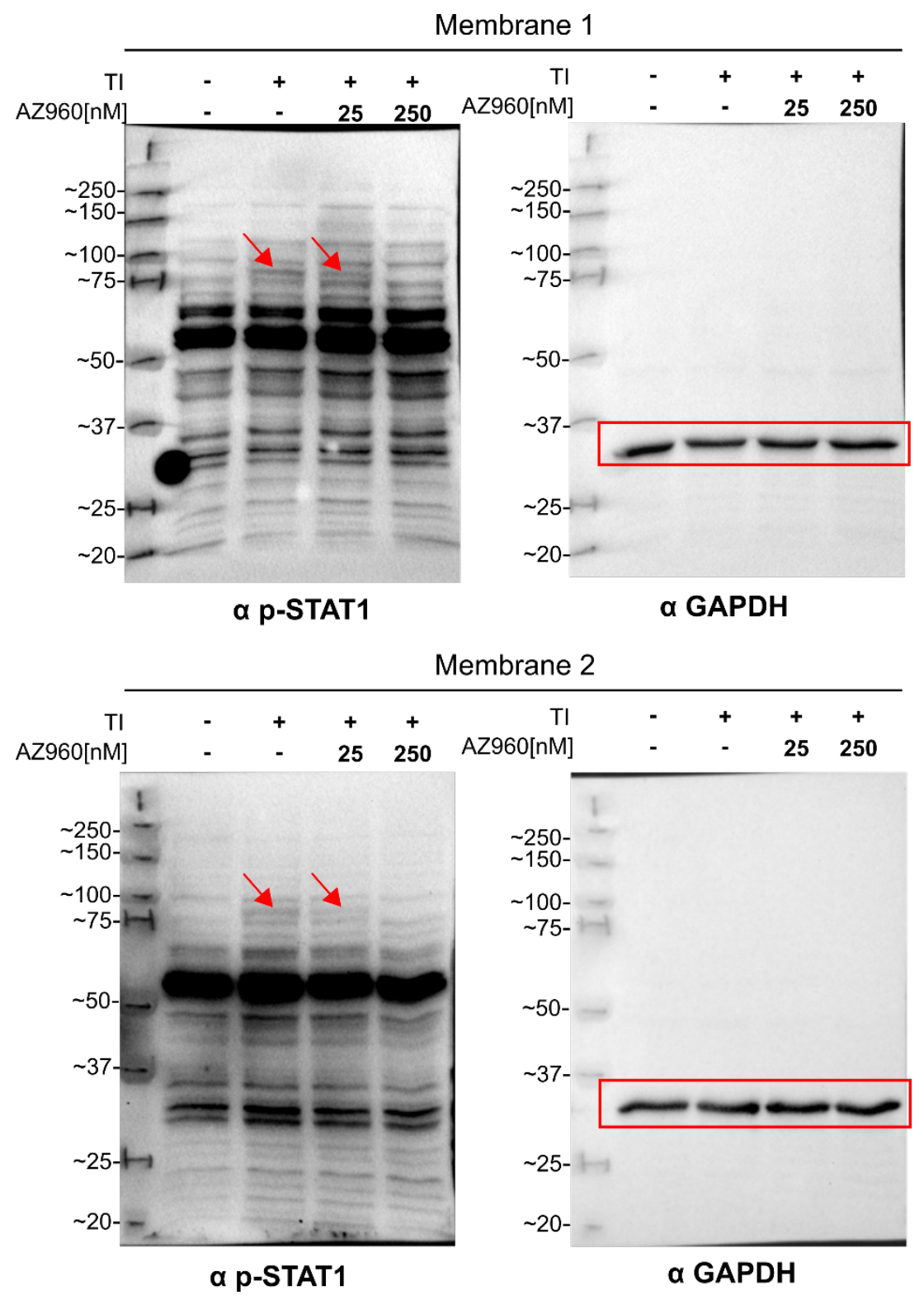
C. Total STAT3

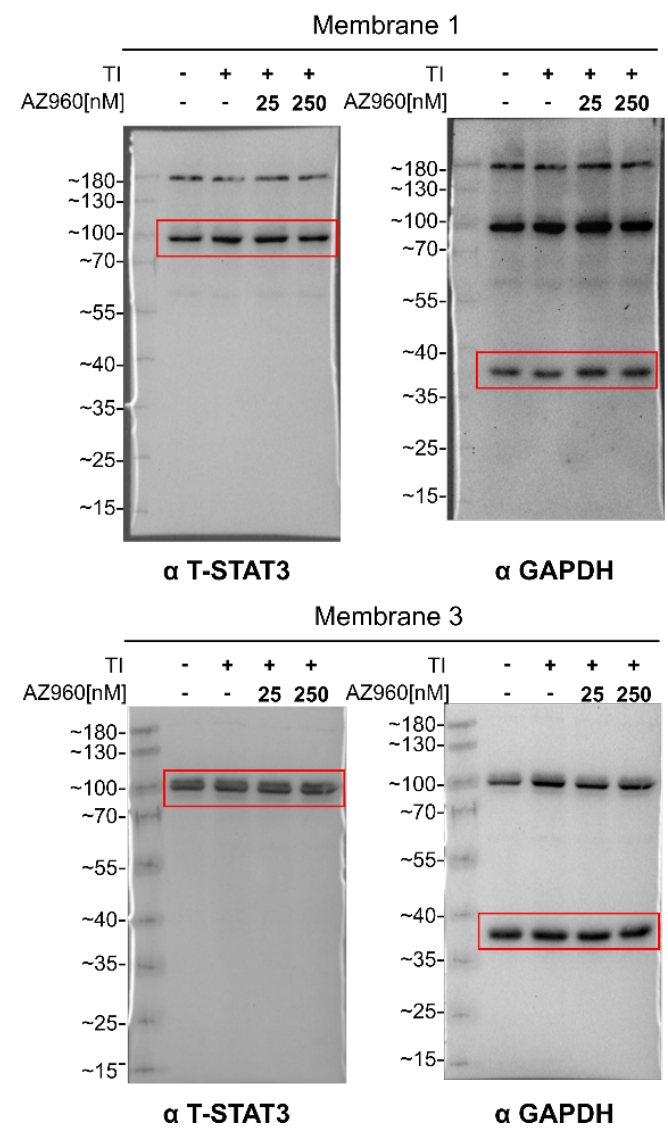

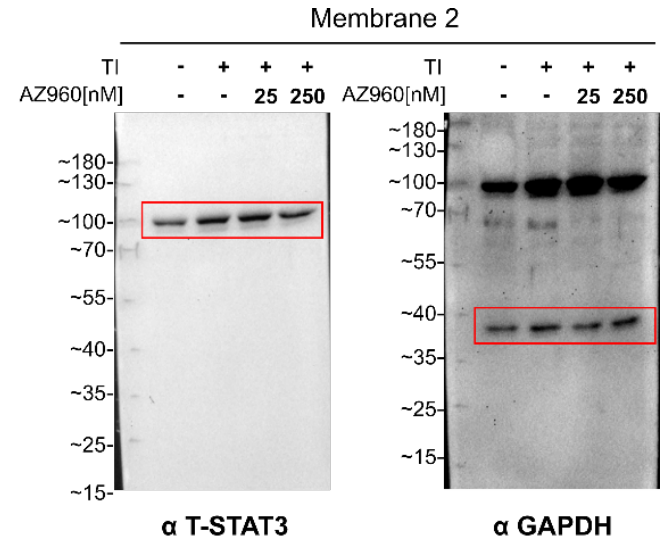


D. p-STAT3

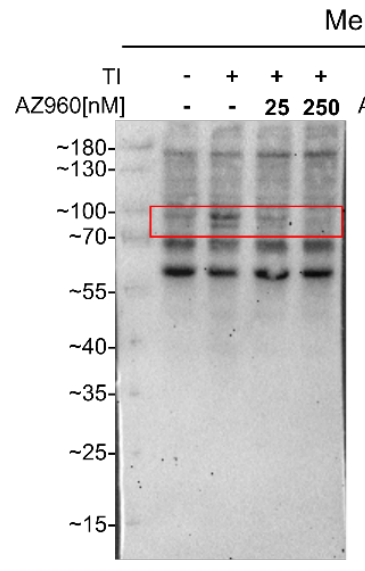

a p-STAT3
Membrane 1

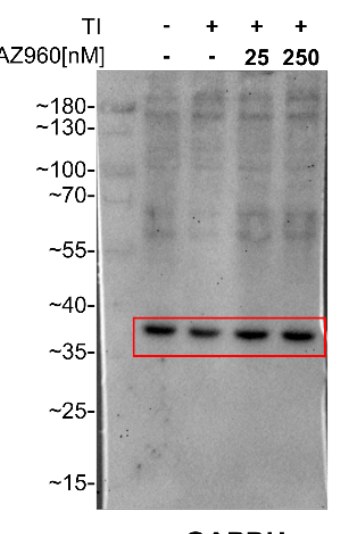

a GAPDH

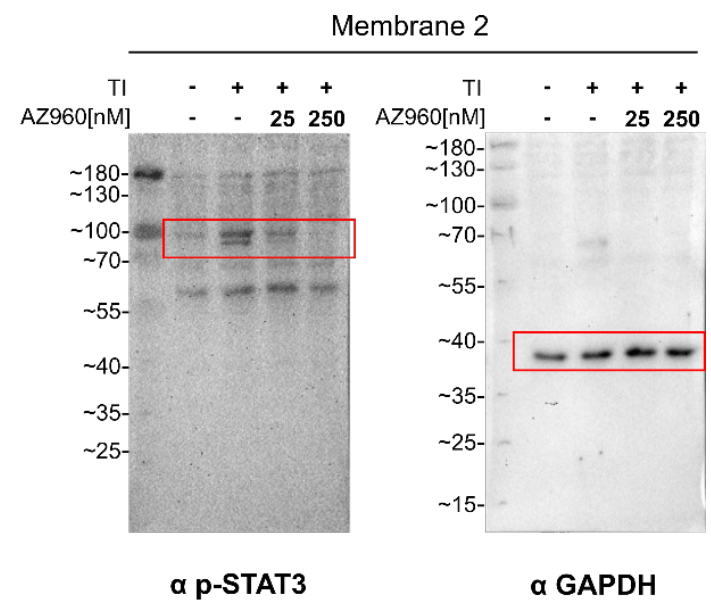

Membrane 3

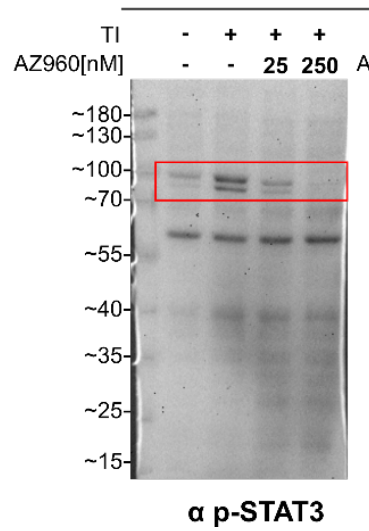

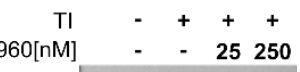

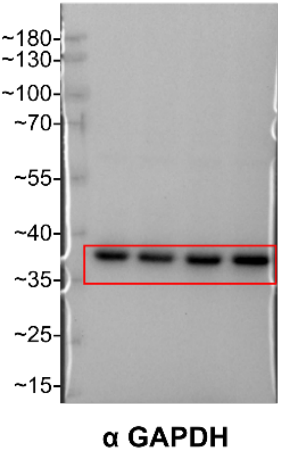


E. Total STAT5

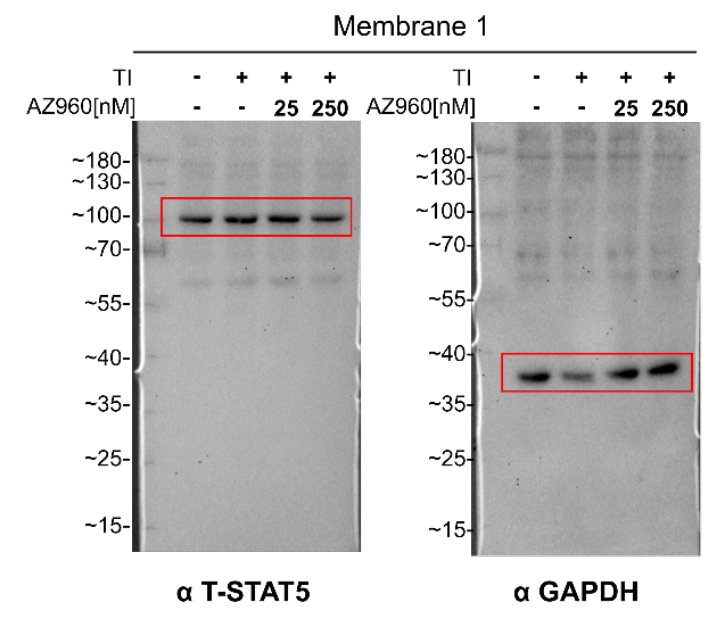

Membrane 3

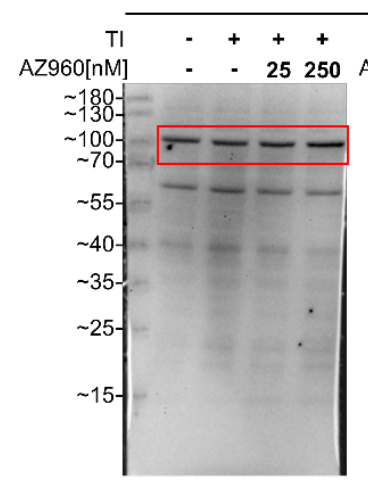

a T-STAT5

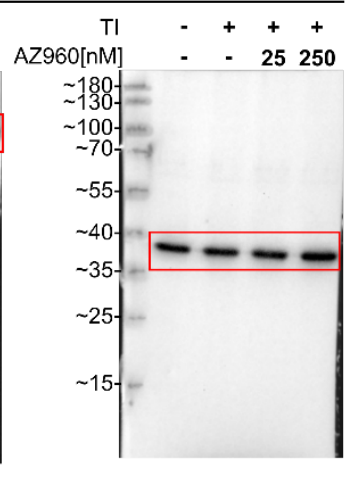

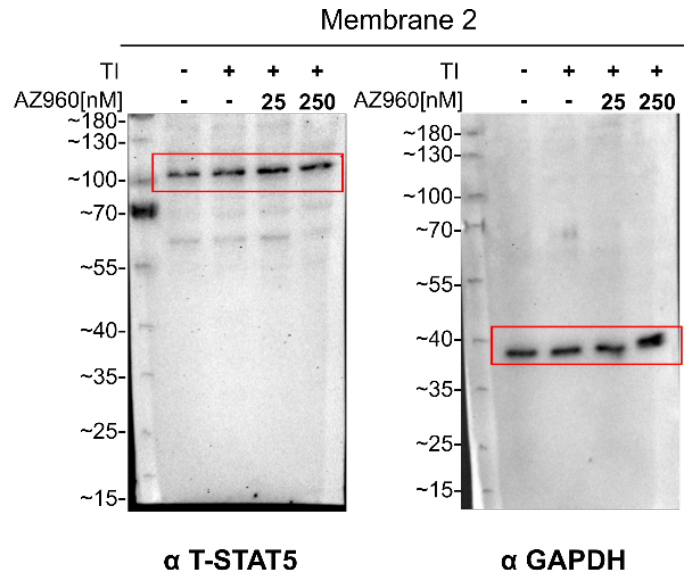

$\alpha$ GAPDH 
F. p-STAT5
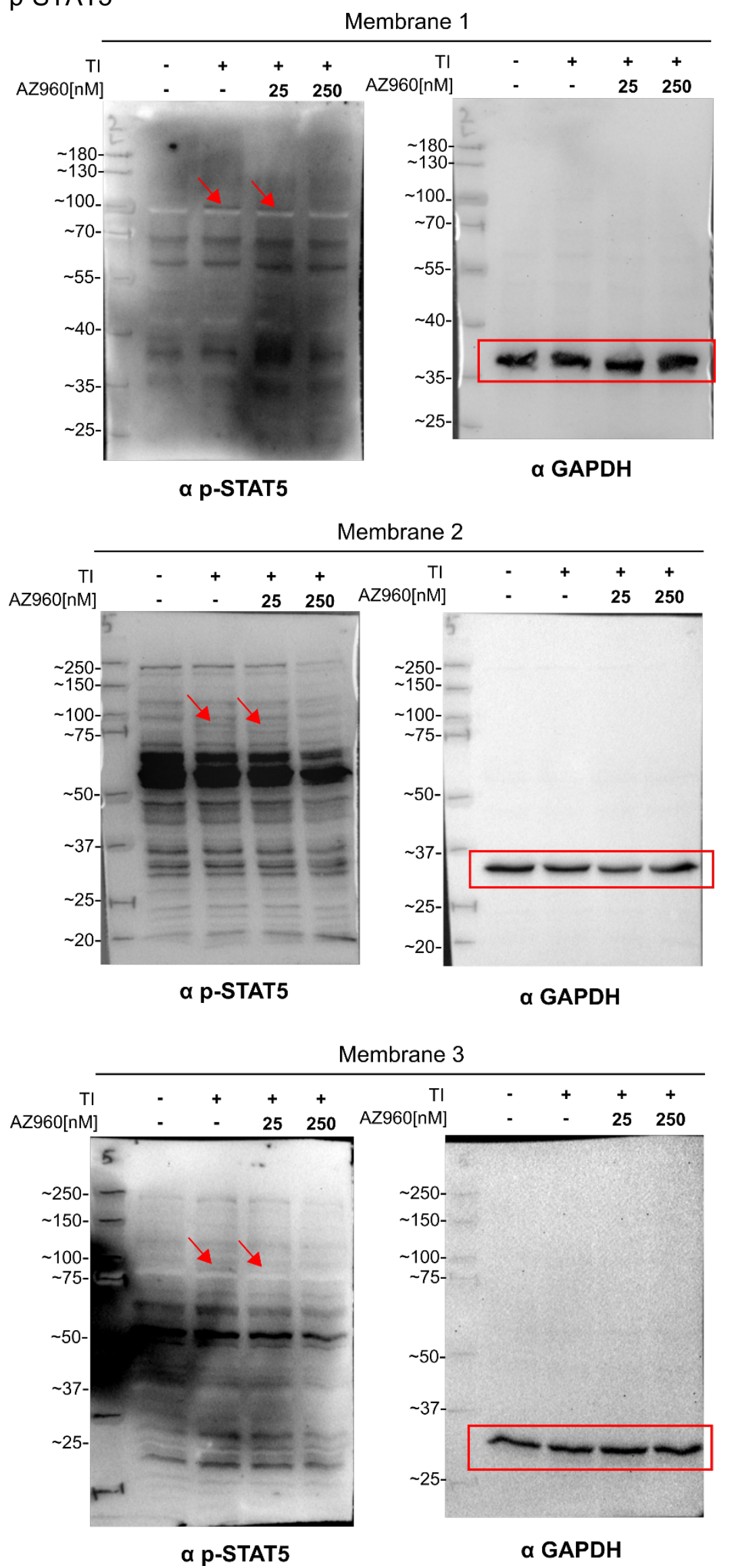
G. FAT10
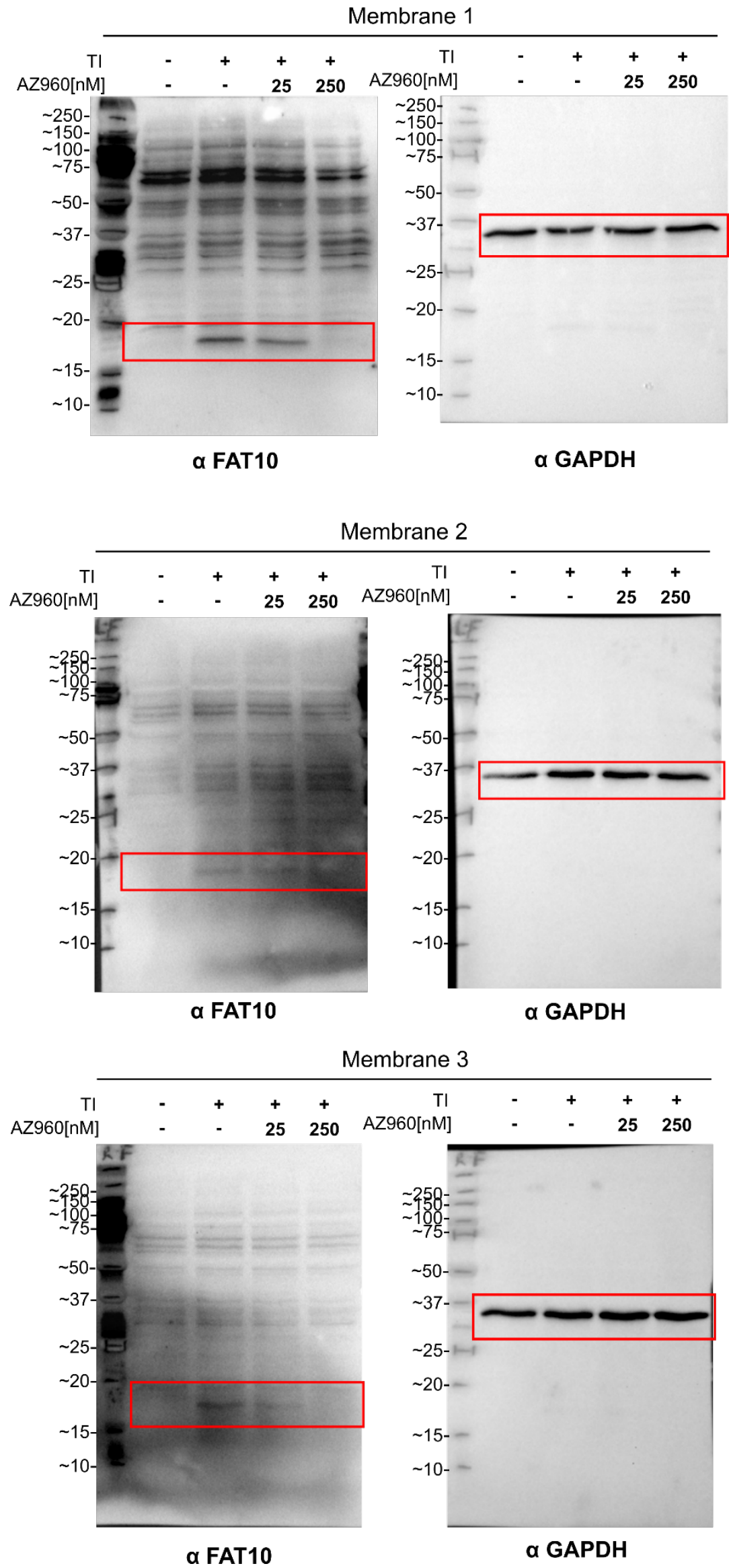


\title{
Supplementary materials and methods
}

\author{
FAT10 promoter region - \\ $\underline{\text { Underline }}=$ FAT10 promoter region cloned \\ Blue = exon, colord according to RefSeq NM_006398.3 \\ Red ATG= transcription start
}

>chr6:29527401-29529800

TCCAGATCTCATGTTGAAATGTGACCCCCAGTGTTGGAAATGGGCCCACTGGGAGATGTTTGGGTTGTG GTGGCAGATCCCTCATGAATGGCCTGGGGCTGTCATCATTGTAATAAATGAGTTCTCACTCCATTAGCT CACAAGAGAGCTGGTTGTTTAAAAGAGCCTGGCATTTTTCCCACTGTCTCTTACCTCCСTCTCTTGCCA TGTGACACGCCTGCTCTCCTTCACCTTCCACCATGAGTAAAAGCTTCTTGAGGCCTCACCAGAAGCAGA TGCTGGTGCCATACTTCTTGTATAGTTGGAGGAACTATGAATCAATTAAACTTTTTTTCCTTATAAATT ACCCAGCCTCAAGTATTCCTTTATAGTATGCAAAAGAGACTAAAACAGCAAATGAAAAGAGATCTGTCT CTGAAAGAACTTATTGTGACATAGAGAGAGACAGAAAAAAGTCAGATTGACACTGGGTAAGAAGCAAGG AGGTCAGGTCCCAAGATGAAGTCCTGCCTGTGGTCAGCAAAGGGGCACCAAGGTGTCTGGGACAGTCCT GGCCCTGGCTTTGGGCAGGGAGGGAATTTCCCATAGGAAGGGAAGAGTAAAGAGAGAGAGAGAGGTCAG AGTCCAGGTTTGTTGTTCATATGTTTTCTTGAAAGGGCACTATTTCCCAGAATCCAGGTCATCTCTGGG TAGGGAATCCCCTGAATTAGTTTTTTCTGAGAGTATATTAGATTTGCCCTCACAGTAACCCCATGCTGG AAATACCCAACTCCAGTAGCACTTTTCTCCCTGCCTTTTCCCACTGAAATAACATAATAATATAGGAAG AATACAAGGACTATAGAAATACAGATTAGTGTTTGAACCCTTGCTTACCAGCTACTACTAATATGATTG TGGATGAGGTAGCTTCTTACTTATTAACGGGGATACTAATAGAGGTGGTTCCTTACAATGATTCCATTT ATGATTTTTTATTTAATAGTGATACCAAAGCAATACATACTCAGTAGAAACCTACTTCAAGTTCCCATA AAATCATCTGCTTTTCACTTTCAGTACAGTATTTAATAACTTAAATGAGATATTTCACACTTCAGTAGT AAATACACTTTTTGTTAGATAATTTTGTCCAACTGTATGCTAATGTAAGTGTTCTGAGCATGTTTAAGG CAGGTTAGGTTAAGCTATGATGTTTGGTGGGTTAGGTGTATTAAATGCATTTCTGATTTTGGATATTTT CAGTGTACAATGGGTTTACAGGGATGTAACCCCATCATAAGTGAAGGAGCACCTGTACTTACTTCATTA AAATGCTGAAACAGTAAATAAGGTAACATTTAATAATATGTTGTGCAGTTCTTGAAATTTAAGTACTCA CCAAATATTACTTTTCCTTTTTTTGTTATTTACTTACTTTTCATTCATTTATTAATTCATTTGTGCATT TAGTAAACATTTATAAATTATTTCCTGTGCCTGACAGCATGCTGGAACAGTGCTAAAGATACAAGTTAA TTAAGACACAATCACGACCCCCAAGATTCCTACTCTTTTCTAAAGATTACAGACAAGCAGACGATGCTA TTGTTGAAGAAACATGCTCTGAGAGGCATTTGAAGGAAGTGTAGAGGATAGAAGATGGACACATAACCC AGGATGGGGAGGAAAAGAGTTAGGGAAGGCTTTTTGACGAAGATACTGTTTACACCGTGTGTTCTTATA AATTCATGGTGGTGGGGATAGAGTTGGAGGAAAAGGCATGCTCAGTGGCGTGGAGATGGCAGAGAGATT GGGGTGTTCAAGGATATGCCGGGAATTCAAGGAACGAGAATTCCCATAGACACAGACACAGCTAGACAT AGAGATCTGCAGCTTAGGTTTGGGCTGTGGGTATAGATCCAGGTGGCTTCAACAGACAAAGATCTTTCC TGAGAAAAGGGAAAAGTTTTCAACACAGAAAGACCATCCCATGTTTGGAATGAGGTTTGCAAATAgatt gcttgaggagagaagtatgtgatcagaaagcattctttgtctattaactcctgcccagcaaaagtgaaa gaaattcatgggagcatgcaagaacaaagagcacagcaaagctggacaaacacagcaatccaggcagg ggatttccaactcaactctggtatataagctgcatgcaaagtcctttttctgtctctggtttctggccc cttgtctgcagagatg 
qPCR primers -

\begin{tabular}{|l|l|l|}
\hline $\mathbf{G e n e}$ & $\mathbf{F} \mathbf{5}^{\prime} \mathbf{~} \mathbf{3}^{\prime}$ & $\mathbf{R} \mathbf{5}^{\prime} \mathbf{~} \mathbf{3}$ \\
\hline FAT10 & CCGTTCCGAGGAATGGGATTT & GCCATAAGATGAGAGGCTTCTCC \\
\hline Actin & CATGTACGTTGCTATCCAGGC & CTCCTTAATGTCACGCACGAT \\
\hline GAPDH & CAACGGATTTGGTCGTATTG & GATGACAAGCTTCCCGTTCT \\
\hline GFP & AAGCTGACCCTGAAGTTCATCTGC & CTTGTAGTTGCCGTCGTCCTTGAA \\
\hline NFKBIA & ATGCTCAGGAGCCCTGTAATG & CCCCACACTTCAACAGGAGT \\
\hline IRF1 & AAGTCCAGCCGAGATGCTAA & TAGCTGCTGTGGTCATCAGG \\
\hline TNFAIP3 & GCGTTCAGGACACAGACTTG & TTCATCATTCCAGTTCCGAGTATC \\
\hline IL8 & TCTGGCAACCCTAGTCTGCT & AAACCAAGGCACAGTGGAAC \\
\hline TNF & TGCTGCAGGACTTGAGAAGA & GAGGAAGGCCTAAGGTCCAC \\
\hline JAK2 & TCTGGGGAGTATGTTGCAGAA & AGACATGGTTGGGTGGATACC \\
\hline
\end{tabular}

\section{RF cloning primers -}

Cloning FAT10 promoter from gDNA into pLEX vector.

Forward 5' - 3':

GGACCTTCTAGGTCTTGAAAGGAGTGGGAATTTCCAGATCTCATGTTGAAATGT GACC

Reverse 5' - 3':

CTCCTCGCCCTTGCTCACCATGGTGGATCCCCTCTGCAGACAAGGGGCCAGAA $\mathrm{AC}$ 